\title{
El Rol de la Mujer en la Práctica del Trueque como un Proceso de Comunicación Propia
}

\author{
The Role of Women in the Barter Practice as an Own Communication Process
}

Luz Mery Avirama Calambas ${ }^{1}$

\section{Resumen}

El "rol de la mujer en la práctica del trueque como un proceso de comunicación propia", es una investigación realizada en el Resguardo indígena de Kokonuko, Municipio de Puracé, Colombia, y contó con la participación de mayoras y mayores, jóvenes, niños, profesores, y autoridades del cabildo que hacen parte de esta comunidad. El propósito del estudio fue conocer las vivencias culturales, el sistema de la memoria cultural, y el testimonio vivo a través de entrevistas con representantes de familias de diferentes veredas que pertenecen al resguardo. Este trabajo pretende ser un camino que oriente los procesos educativos y a la comunidad misma. Esta investigación busca fortalecer el ejercicio ancestral de la práctica del trueque como una herramienta de seguridad, autonomía, soberanía alimentaria y territorial mediante la recuperación de prácticas ancestrales de la comunidad, focalizada en el rol que ejerce la mujer en una estrategia que evidencia el proceso de comunicación propia en el resguardo.

Palabras clave: Comunicación propia; trueque; rol de la mujer; semillas nativas; autonomía; soberanía alimentaria; educación.

\section{Abstract}

The "role of women in the barter practice as a communication process of their own", it is an investigation carried out in the Kokonuko indigenous reservation, Puracé Municipality, Colombia, and it counted with the participation of the elderly, young people, children, teachers, and council authorities that are part of this community. The purpose of the study was to know the cultural experiences, the cultural memory system, and the living testimony through interviews with family representatives from different villages that belong to the shelter. This work aims to be a path that guides the educational processes and the community itself. This research seeks to strengthen the ancestral tradition of the barter practice as a tool of security, autonomy, food and territorial sovereignty through the recovery of ancestral practices of the community, focused on the role that women play in a strategy that demonstrates the process of Own communication in the shelter.

1 Dinamizadora de Educación en el Consejo Regional Indígena del Cauca (CRIC), y, Máster en Comunicación Intercultural con Enfoque de Género. E-mail: meryav25@gmail.com

Recibido: 26/02/2018 Aprobado: 28/08/2018 
Keywords: Own communication, barter, women role, native seeds, autonomy, food sovereignty, education.

\section{Introducción}

Esta investigación busca fortalecer el ejercicio ancestral de la práctica del trueque como una herramienta hacia la seguridad, autonomía, soberanía alimentaria y territorial a través de la recuperación de prácticas ancestrales de la comunidad, focalizada desde el rol de la mujer en la práctica del trueque como un proceso de comunicación propia en el resguardo.

También busca revitalizar la conciencia en la comunidad en tanto se constituya como una alternativa comunicativa y económica que fortalece la huerta familiar, comunitaria y escolar. Asimismo, contribuye al rescate de las semillas nativas del resguardo y dinamiza el propósito de la Organización que representa el rol de la mujer en la práctica del trueque mediante la tradición oral como mecanismo de comunicación propia.

La importancia de esta investigación se centra en mostrar que en el intercambio de productos no se utiliza el factor monetario, al contrario, se intercambian conocimientos, ideas, sabidurías, valores culturales e ideologías. Dicho proceso investigativo tuvo el respaldo y participación de la autoridad tradicional, las mayoras, mayores, mujeres, jóvenes, señoritas, niñas y niños de los centros educativos. Esto hizo posible realizar con facilidad la recolección de los datos mediante las entrevistas de campo que se necesitaron para la construcción de este trabajo.

\section{Revisión de literatura}

El Resguardo indígena de Kokonuko, desde la recuperación más conocida como la liberación de la madre tierra, inicia a partir de 1971, con la conformación del Consejo Regional Indígena del Cauca, el 24 de febrero de 1971 (CRIC, 2007). Rastreando archivos históricos que den cuenta de su existencia, están el título primario del Resguardo de Kokonuko otorgado por la corona española en el año 1736. (Lineamiento Plan de Vida Zona Centro, 2008, p.18).

La existencia del pueblo Kokonuko, cuyo nombre significa "Lomas Hondonadas", es narrada por las crónicas de los conquistadores españoles. Formaba parte de los diversos pueblos existentes en el área que hoy comprende POPAYÁN y sus alrededores, tales los Andaquies, Kokonuko Guanacas, Nievas, Paeces, Puraceños, Polindaras, Timanaes Totoroez y Misaks. 
En el Resguardo de Kokonuko la mujer siempre ha tenido el espacio de comunicación propia, donde puede transmitir sus saberes, pensamientos, prácticas culturales, ancestralmente ha utilizado la palabra a través de la tradición oral.

Frente a este significativo hecho, la mujer juega un papel fundamental y decisivo en la batalla por la recuperación de las tierras. Hoy, hace parte de las estructuras político organizativo ancestral de la comunidad de Kokonuko, trabajan colectivamente en la estructura del cabildo, en los programas de comunicación, salud, educación, mujer, medio ambiente, producción, y participan en la guardia indígena, entidad encargada del control territorial y social.

\section{Significado cultural del Pueblo Kokonuko}

Los Kokonukos se encuentran asentados en una región provista de una infinita gama de verdes y abundante agua, rodeada de volcanes entre ellos Puracé y Sotara que da el nombre al municipio donde se encuentra el resguardo del mismo nombre. Este pueblo cuenta en su territorio, con aguas termales, una mina de azufre empotrada en las montañas de la cordillera central y aguas saladas en el resguardo de Poblazón. Su organización sociopolítica, fue una de las primeras en ser desestructurada a través de la figura de la encomienda, durante la colonia, en 1559, o sea 67 años después de la llegada de Cristóbal Colón, con el fin de usurpar sus tierras.

Cuentan los mayores que los Kokonucos provenimos de dos grandes Espíritus, los volcanes Jukas y Sotará que a través de bolas de fuego fecundaron el territorio con lava de sus entrañas. La madre naturaleza era entonces de paramos, lagunas, ríos, riachuelos, que bajan de la gran montaña sagrada Kokonuko que es el Espíritu Mayor de cabeza brillante. Los nevados se comunicaban unos con otros a través de surcos de fuego que llegaron a explotar en el centro de la cordillera provocando una gran avalancha trayendo consigo la Serpiente que se quedó en la meseta y luego se transformó en otras formas de vidas como la Mamá Dominga y los caciques que dieron origen al Pueblo Kokonuko. (Lineamiento plan de vida zona centro, 2008.pag.18).

La comunidad conserva el principio de la colectividad lo cual se refleja en la minga, el cambio de mano y la participación en consenso de sus determinaciones a través de las asambleas, hoy los congresos, juntas directivas, encuentros de saberes; creemos en los espíritus que armonizan nuestras relaciones con la madre tierra como el duende, el guando, la viuda, y otros espíritus de energías positivas o negativas; los sueños, las señas corporales, los mensajes de la naturaleza que transmiten aves, serpientes, sonidos, nubes, y las formas de la luna que anuncian acontecimientos. 


\section{La Educación y estrategias pedagógicas}

A fin de contribuir a la formación educativa de la comunidad se viene desarrollando la educación en el marco del sistema educativo indígena propio SEIP, como iniciativa del Consejo Regional Indígena del Cauca (CRIC). Durante el V Congreso llevado a cabo en el resguardo se reafirma el derecho que tienen los pueblos indígenas con la recreación y fortalecimiento de las lenguas originarias, usos y costumbres a partir de la escuela, sin dejar de lado el entorno en que los niños y niñas se desenvuelven. En este contexto nacen y se fortalecen las prácticas del trueque estudiantil como modelo pedagógico desde el cual se fortalecen la unidad, la solidaridad y la sostenibilidad de la comunidad.

Las dinámicas pedagógicas de algunos resguardos han permitido dinamizar el trueque entre instituciones educativas como estrategia de fortalecimiento del proceso cultural de nuestro pueblo, con el objetivo de ir sembrando en las niñas y niños el sentido de pertenencia y arraigo cultural especialmente en este ejercicio. Es ahí donde el proceso educativo juega un papel importante, incluyente y articulador de las dinámicas comunitarias a los espacios escolares o educativos apoyados por los docentes, padres de familias y equipos de educación.

La importancia del trueque radica en su carácter educativo, lo que conlleva a la implementación de actividades que fortalecen la unidad mediante el intercambio de productos y saberes enraizados en la familia, la comunidad y mayores. Esto asegura que las experiencias y vivencias fortalezcan procesos educativos permanentes con el propósito de arraigarlas como formas de vida cultural.

\section{Comunicación, identidad cultural y resistencia}

Comprender la comunicación desde el pensamiento propio de los pueblos originarios es poner en perspectiva la cosmovisión e identidad que la fundamenta y le da sentido a las prácticas ancestrales como principios de vida comunitaria. Es una comunicación que se define milenariamente, desde la familia, la comunidad, la humanidad y el universo, como vía de interrelación y medio espiritual de estrechar lazos con la madre naturaleza.

La comunicación se produce en contextos difíciles de los pueblos indígenas sobrevivientes del exterminio vivido con la colonización. Ello propicia un proceso de reflexión en torna a la comunicación como una herramienta estratégica de resistencia en la lucha y en la construcción de la autonomía de los pueblos. (Cumbre Continental de Comunicación Indígena de Abya Yala.pag.11). 


\section{La Tradición Cultural del Trueque}

Para el pueblo Kokonuko, el trueque es una tradición cultural y ancestral practicada por las familias y consiste en el intercambio de productos alimenticios y medicinales incluyendo los saberes ancestrales, que surgen en la familia, la comunidad y mayores.

Así lo manifiesta el mayor Julio Hernández, Comunero del Resguardo de Kokonuko, (entrevista personal, 25 de mayo del 2016).

“(...) para mí el trueque es una práctica que deja enseñanzas, en él se transmite y se aprende desde la oralidad a la práctica, de la mano con nosotros los mayores, la familia y la naturalez, es un proceso que se dinamiza en el territorio, en la experiencia del diario vivir. La gran importancia del trueque es cuando llevamos a la práctica el conocimiento convirtiéndose en un saber".

El trueque se da como un proceso de vida, involucra conocimientos, habilidades y sentimientos. Su sentido y significado radica en la capacidad de articularse como individuo en un colectivo y sentirse participante de un proceso integral. Es una práctica intercultural porque reconoce y valora las diferentes culturas que conviven en el territorio en donde se establece una relación de diálogo y entendimiento que orienta la formación respetuosa en una relación de convivencia armónica, y fortalece lo propio como legado cultural.

El trueque adquiere su significación al potenciar los diferentes pisos térmicos, el saber tradicional agrícola y las estructuras organizativas del movimiento indígena. Conscientes de estas riquezas, los indígenas convierten al trueque en otra expresión de resistencia en contra de las políticas económicas. Más exactamente contra el TLCy el ALCA. "trueque en tiempos de globalización" es su lema. O como dicen los indígenas del putumayo: "de la chagra a la resistencia".

El trueque va a acompañado de un trabajo de sensibilización y capacitación a la comunidad en seguridad y autonomía alimentaria, derechos humanos, coyuntura política y cultural. Se les denomina "encuentro de pensamiento, lucha y reivindicación de resistencia". Para las autoridades tradicionales, "estos encuentros se realizan contra las cosas que nos imponen el gobierno para responder como organización indígena" o "demostrarle al estado que estamos organizados y que seguimos resistiendo". (Galeano, 2006, p.290)

Hoy en día y gracias al trabajo de la organización del Consejo Regional Indígena del Cauca (CRIC), la Asociación de Cabildos "Genaro Sánchez" y las comunidades de los resguardos de la zona centro que retomaron esta dinámica a nivel zona, se ha logrado practicar entre resguardos, ya que el territorio del pueblo Kokonuko tiene productos de todos los climas posibilitando la recuperación y dinamización del trueque. 
Anterior a este empuje, el trueque como impulsor de la economía propia, la soberanía alimentaria, la identidad cultural y el sentido de pertenencia, habían sido debilitados hasta casi desaparecer. Debido entre otros factores, el ingreso de personas externas al territorio, el impacto de las políticas económicas capitalistas, determinantes sociales, políticos, religiosos y educativos.

\section{La revitalización del trueque como una práctica ancestral e intercultural}

En el Pueblo Kokonuko se toma la decisión de recuperar el trueque por orientación de los mayores, como una práctica ancestral, un camino de conocimiento y sabiduría. De esta manera se intercambian productos agrícolas y pecuarios, se presentan actos culturales con música de chirimía, danzas, se relacionan e intercambian saberes, se fomentan prácticas de conservación de semillas propias y plantas medicinales, y, tradicionales culturales.

Los trueques son programados según el tiempo de cosecha tanto en la parte media, cálida y fría con el fin de que todos salgan beneficiados. Por ello, cada cabildo participante subsidia el transporte y asegurar asistencia masiva. De esta manera, es una práctica intercultural porque reconoce y valora las diferentes culturas que conviven en el territorio en donde se establece una relación de diálogo, entendimiento y convivencia armónica.

\section{Materiales y métodos}

\section{Estrategia de Comunicación Propia para Caminar desde la Práctica del Trueque}

Encontrar el camino estableciendo líneas de estratégicas desde la comunicación, enmarcadas por el proceso organizativo de los pueblos partiendo de sus realidades, abarcando desde la resistencia hasta la autonomía de la palabra y la vida colectiva. Dentro de estas estrategias a seguir esta el fortalecimiento del trueque enfocado desde el rol de la mujer, como un elemento de comunicación propia, identificando opciones para conseguir conjuntamente políticas de comunicación diferencial para la comunidades indígenas articular y transformar en acciones y compromisos puntuales a nivel local, regional se debe entender la comunicación desde la espiritualidad desde la resistencia y lucha que seguimos y desde los desafíos que enfrentamos, caminando hacia la construcción en unidad de la palabra colectiva.

Con el avance de la tecnología y que poco a poco ha ido invadiendo nuestro territorio con medios de comunicación, analizando esta situación, se toma la decisión como pueblo Kokonuko en crear y apropiarse de la emisora propia comunitaria, como mecanismo de difusión de nuestros procesos y el fortalecimiento cultural. En el contexto familiar del resguardo de Kokonuko, la mujer es la que constituye la parte 
estructural y fundamental del hogar, es desde este espacio donde podemos ver que se transmite las tradiciones, valores culturales, amor, solidaridad, el respeto pero en si también se fortalece y recrea la mantención del trueque como uso milenario.

\section{Resultados y discusión}

El Rol de la Mujer en la práctica del trueque es un pilar fundamental como lo comenta la señora Sandra Calambas y alejandrina jalvin en este proceso organizativo de nuestra comunidad; son las portadoras de mensajes, brindan orientaciones y espacios de Integración, comparten conocimiento de saberes ancestrales propios, que son maneras de comunicarse, transmitir información, sentimientos, opiniones. Permitiendo fortalecer la comunicación a través de mensajes y símbolos con la naturaleza, las tradiciones usos y costumbres de la comunidad para continuar perviviendo como Resguardo indígena de Kokonuko en el tiempo y en el espacio. Una de ellas "EL TRUEQUE”.

Como nos lo comenta la compañera Sandra Calambas, Comunera del Resguardo de Kokonuko. (Entrevista personal, 10 de diciembre del 2015).

Personalmente creo que las mujeres somos las portadoras de todo el conocimiento porque somos las que picamos, sembramos, cosechamos, y enseñamos como se hace la labor en el campo, somos aquellas que le transmitimos a nuestros hijos el conocimiento que nuestros papas nos han dejado porque ellos se esforzaban mucho para que nosotros aprendiéramos a valernos con cada planta que sembráramos y también ellos mismos nos enseñaban el valor que tenía todo lo que hacíamos no solo era sembrara por sembrar todo o teníamos que hacer con amor que lo sintiéramos por ellos nos decían que si sembrábamos con amor así mismo se iban a dar las plantas porque ellas sentían igual a nosotros. Además nosotras somos las primeras que nos levantamos y las ultimas que nos acostamos porque somos las que orientamos en todos los campos y como decía mi abuelo Jorge ustedes mis viejas son el pilar de nosotros que sería de la vida sin ustedes así sea cascarrabias pero ustedes hacen mucha falta.

Claro que sin dejar a un lado el rol del hombre en estos espacios. Pues hablándolo así ellos en un tiempo eran muy egoístas a dejar que las esposa o compañeras participaran en estos espacios o actividades ahora las cosas ya han cambiado ellos participan de algunas actividades pero ya le dan como el espacios a las mujeres para que aprovechen estos espacios, en algunos hogares o familias por llamarlo a si ellos les ayudan a sus mujeres a cultivar la tul ósea la huerta son ellos que también están pendientes de las o plantas personalmente lo hablo por mi marido el me ayuda a cultivar mi huerta y juntos participamos de los trueques porque siempre que vamos 
a estos espacios intercambiamos todo lo que llevamos además también intercambiamos sabidurías y conocimientos, experiencias conseguimos amistades y nos gusta ya mi hija también le gusta practica más que el conocer le gusta el intercambio porque me comenta que ella aprende mucho de estos espacios y entonces uno mira es que lo que no se coloca en práctica es lo que no se hace por que anteriormente las mujeres éramos muy sumisas no salíamos del fogón por miedo a nuestros maridos pero las cosa hoy en día han ido cambiando ellas son las que tejen la sabiduría en estos espacios. (Calambas, 2015).

Una de las funciones que ejerce la mujer en las parcelas y huertos familiares, desde este espacio expresa conocimientos, valores tradicionales desde la teoría y práctica, además es quien se dedica a ser la Custodia de Semillas (persona que se dedica a guardar o conservar las semillas), quien respetuosamente guarda con cuidado las semillas, mantener la soberanía alimentaria, seguridad alimentaria y biodiversidad en nuestro territorio ya que es una forma de comunicación propia, además es la persona que se encarga de mantener la tradición al interior de la familia impartiendo conocimiento a los hijos.

\section{Entretejido del trueque como proceso de comunicación propia}

En la comunidad indígena de Kokonuko tenemos encuentros de saberes donde se refleja nuestra tradición colectiva como La Minga, el cambio de mano, las asambleas, congresos, Juntas directivas, comunicación, encuentros de juegos tradicionales, armonización y relación con la madre tierra, la naturaleza, la luna que anuncian acontecimientos.

En el del trueque es una práctica cultural ancestral, que consiste en intercambiar conocimientos, saberes, reflexiones, comunicación, semillas y también productos, cultivados en los distintos territorios teniendo en cuenta los climas, para esto es muy importante hacer partícipes a los territorios donde haya variedad de producción agropecuaria esto con el fin de que la práctica sea más dinámica y que las comunidades participantes se lleven un excelente intercambio. En este se tiene un proceso de comunicación propia desde la programación, el sacar la fecha en que resguardo se va a realizar, la logística, quienes participan. Los padres y madres comparten con sus hijos en la recolección de productos, en la preparación para participar. En el trueque se conversa, comparte experiencias, saberes, conocimientos, semillas, artesanías, danzas, también el yo te enseño y tú me enseñas, se distrae, se recrea, se deleita de comidas propias tradicionales, intercambio de productos sin el uso de dinero, basados en las necesidades de cada comunero o familia, donde se Intercambian productos de clima frio y cálido entre otros. 
En esta práctica cultural y ancestral participan niños, niñas, jóvenes, jovencitas, mayoras, mayores, Autoridades Tradicionales y la guardia indígena a través de saberes culturales, como danzas, canciones, coplas, alusivas al trueque, exposición e intercambio de semillas y productos que se tienen en cada uno de los diferentes Resguardos del Pueblo Kokonuko, que es una forma de fortalecer el proceso de nuestra comunicación propia. Esta práctica Ancestral, se retomó en la zona centro, Pueblo Kokonuko en el departamento del Cauca desde el año 2003, se revitaliza y fortalece esta práctica con la guardia Indígena, se realizó el primer trueque de semillas y productos en el Resguardo del Alto del Rey, mirándolo y analizándolo como una alternativa o estrategia de resistencia ante el capitalismo y globalización del mercado; buscando resolver las carencias de alimento, vestido, salud, educación, vivienda entre otros que el modelo económico mercantilista actual ocasiona. La resistencia y la economía, se amplían por todos los participantes en cada uno de los encuentros, quienes ratifican en sus líderes el compromiso en la construcción de un proyecto de vida ajustado a sus condiciones geográficas, sociales y culturales, a fin de trasmitir a las nuevas y futuras generaciones posibilidades de permanecer como indígenas.

\section{En Minga Sembrando Saberes desde la Huerta Escolar}

En Minga sembrando saberes desde la huerta escolar: cuando hablamos de la minga queremos decir que es el ejercicio que se practica para el desarrollo de actividades ya que esto nos genera una economía comunitaria y nos hace que las labores rindan un poco más, se parte que es un espacio donde se siembra, se cultiva las prácticas culturales, conocimientos de nuestras mayoras y mayores; también es donde se realiza o se desarrolla trabajos para el fortalecimiento de la autonomía, soberanía y seguridad alimentaria, conservando y protegiendo nuestra tierra, además es una herramienta pedagógica para articular las áreas.

Las niñas cumplen un papel fundamental en el proceso del trueque estudiantil porque ellas son las que organizan, retoman las experiencias de vida de los padres y abuelos, haciendo un alto en el camino donde reflexionan a través de mingas de pensamiento, para trazarse metas brindándole a su comunidad estudiantil oportunidades para que se integren en el proceso de aprendizaje, formación, que contribuye a valorar y fortalecer el desarrollo de una comunicación propia y de una educación para la vida.

Los establecimientos educativos tienen terreno para la siembra, se hace la limpieza, se pica y se abona. Luego se implementan las siembras en el tiempo indicado, orientado por las mayoras y mayores, estas siembras se hacen ya sean de zanahoria, remolacha, acelga, cilantro, repollo, arveja, cebolla, coliflor, habas, entre otros. En la huerta escolar también se puede cosechar desde todo lo que aprendieron los estudiantes a través de la articulación de las áreas del conocimiento (Matemáticas y Producción, Comunicación y Lenguaje, Comunidad y Naturaleza, Territorio y Sociedad) del Proyecto Educativo Comunitario (PEC). Que para el caso de la zona centro, es la Jigrapucha del 
PEC del Pueblo Kokonuko. En esta se crean estrategias didácticas desde los proyectos pedagógicos en el marco de la Jigrapucha del PEC del Pueblo Kokonuko. Desde este espacio se da el desarrollo de dinámicas de trabajo integrado por estudiantes, padres de familia, comunidad, autoridad tradicional y dinamizadores comunitarios y docentes. Los estudiantes aprenden a investigar, el hacer, construir, compartir, unidad, donde las vivencias son el soporte para fortalecer las costumbres y la identidad.

La Jigra es un proceso de tejido cultural milenario, a cargo de nuestras mayoras y mayores, consiste en construir caminando la jigra, existen unos momentos de elaboración el primero lo llamamos puente que es el asiento, el segundo la primera parada esta determina el tamaño, el tercero es el cuerpo que le da forma, cuarto la cumba y la cincha o cargadera determina la cantidad de peso a cargar. La Pucha no está terminada sigue en proceso de construcción. La Jigrapucha del PEC del Pueblo Kokomuko aún está en proceso de construcción.

\section{Conclusiones}

Con la tarea de seguir contribuyendo en el desarrollo de las prácticas culturales del pueblo Kokonuko, en asamblea con el cabildo la guardia, docentes y dinamizadores comunitarios, padres de familia, niños, jóvenes, señoritas, mujeres, realizan un proceso para analizar las dificultades, avances y proyecciones sobre cuáles son los resultados del trabajo que se viene realizando con el rol de la mujer en la práctica del trueque. Con esto se busca la formación y seguimiento en el contexto, la cultura, la investigación, el espacio de aprendizaje, habilidades, responsabilidades que generan las personas que participan de esto espacios con los compromisos de integración, respeto por la Madre Naturaleza, el Territorio, conservación ambiental ya que estos son espacios de conocimiento que están ligados a las prácticas ancestrales, cotidianas comunitarias.

Abordando la investigación encontramos una comunidad que refleja elementos importantes como la comunicación propia encaminada desde la práctica del trueque donde la mujer es el pilar fundamental, desde este espacio se transmite sabiduría, conocimiento, de igual manera ha servido para solventar el factor monetario. Sin embargo es de anotar que a través de esta práctica ancestral milenaria, un grupo de mayores han formado los custodios de semillas quienes son los encargados del rescate y fortalecimiento de las semillas nativas propias, esto permitiendo fortalecer los procesos organizativos en los espacios dentro de la comunidad.

Se observó que hay padres de Familia, maestros comunitarios, estudiantes, mujeres, autoridades, comuneros de la comunidad que aseguran estar interesados en seguir participando en la práctica del trueque que ha mejorado en cuanto al fortalecimiento de la cultura ya que el grupo de trabajo ha apoyado este proceso, además propenden el mantenimiento de fortalecer la participación propia de cada una de la mujeres. 
El pueblo ha podido aportarle a la creación de propuestas para los centros educativos, lo que evidentemente hace pensar un alcance positivo, que ha tenido la propuesta de la comunidad a organizarse y a educar a sus hijos. La educación que les servirá para el futuro, no solo como estrategia política, social, económica y cultural si no como una forma de entender la realidad del rol de las mujeres indígenas y el resto de la comunidad.

Por lo tanto esta investigación incidió de manera positiva puesto que el trabajo con la autoridad, la comunidad en general y la comunidad educativa ha aportado a mejorar el rol de la mujer en la práctica del trueque como un proceso de comunicación propia. Los grupos que apoyaron el trabajo investigativo continúan participando para seguir fortaleciendo el trueque dentro de la comunidad. Por tal razón vemos importante. En este sentido la socialización de los resultados de la investigación en los espacios de las asambleas comunitarias como también es importante reflexionar en el espacio educativo.

\section{Lista de referencias}

CRIC (2007). Plan de Vida de los Pueblos Indígenas del Cauca. Consejo Regional Indígena del Cauca. Recuperado en: observatorioetnicocecoin.org.co/files/Plan\%2ode\%2O vida\%2odel\%20Cric.pdf

Galeano, M. (2006). Resistencia Indígena en el Cauca: labrando otro mundo. Consejo Regional Indígena del Causa (CRIC).

Lineamiento Plan de Vida Pueblo Kokonuko Zona Centro, 2008.

MUGARIK GABE (2010). Cumbre Continental de Comunicación Indígena del Abya Yala. Encuentros y Jornadas, Latinoamérica, Pueblos Indígenas. Recuperado en: http://www.mugarikgabe.org/blog/2010/10/18/cumbre-continental-de comunicacion-indigena-del-abya-yala/ 